\title{
Sarcoma de Kaposi em pacientes HIV: novamente uma realidade
}

\author{
Kaposi 's sarcoma in HIV patients: a reality again
}

Sarcoma de Kaposi en pacientes VIH: nuevamente una realidad

Sérgio Alberto Lando Borges ${ }^{1 *}$, Lucia Helena Gonzales Real ${ }^{1}$, Renata Backes Schreiner ${ }^{1}$.

\begin{abstract}
RESUMO
Objetivo: Revisar na literatura o Sarcoma de Kaposi em pacientes com HIV/SIDA, descrevê-lo, apontar sua clínica a fim de auxiliar no diagnóstico e apresentar as opções de tratamento. Método: O resumo foi realizado a partir da análise de artigos científicos, selecionando aqueles com conteúdo diretamente ligado ao tema. Resultado: o Sarcoma de Kaposi foi descrito pela primeira vez em 1872, como um tumor mesenquimal, angioproliferativo com infiltrado inflamatório causado pelo vírus herpes humano 8, caracterizado por lesões nodulares hiperpigmentadas. Hoje, é considerada a principal malignidade relacionada a Síndrome de Imunodeficiência Adquirida, e, por poder acometer qualquer sistema do corpo, pode comprometer de maneira significativa a qualidade de vida do portador do sarcoma. Considerações finais: O Sarcoma de Kaposi é uma neoplasia pouco prevalente, porém, com o aumento da infecção pelo HIV diagnosticada tardiamente, vem ganhando espaço no sistema de saúde, sendo necessário saber o identificar, uma vez que o diagnóstico e o tratamento precoce podem diminuir a morbimortalidade das pessoas com HIV.
\end{abstract}

Palavras-chave: Sarcoma de Kaposi, HIV, Sindrome da imunodeficiência adquirida (SIDA).

\begin{abstract}
Objective: To review the scientific literature about Kaposi's sarcoma in HIV / AIDS patients, to describe it, to point out their clinic in order to help the diagnosis and submit the treatment options. Method: The abstract was based on the analysis of scientific articles, selecting those that content directly related to the theme. Results: Kaposi's sarcoma was first described in 1872 as a mesenchymal, angioproliferative tumor with inflammatory infiltrate caused by the human herpes virus 8 , characterized by hyperpigmented nodular lesions. Today, it is considered the main malignancy related to Acquired Immunodeficiency Syndrome and, because it can affect any system of the body, can seriously compromise the quality of life of the sarcoma carrier. Final considerations: Kaposi's sarcoma is a neoplasm that is not very prevalent. Although, with the increase of late-diagnosed HIV infection, it has been gaining space in the health system, and it is necessary to know how to identify it, since diagnosis and early treatment may morbidity and mortality of people with HIV.
\end{abstract}

Keywords: Kaposi's Sarcoma, HIV, Human Immunodeficiency Syndrome (AIDS).

\section{RESUMEN}

Objetivo: Revisar en la literatura científica el Sarcoma de Kaposi en pacientes con VIH / SIDA, describirlo, apuntar su clínica para auxiliar en el diagnóstico y presentar opciones de tratamiento. Método: El resumen fue realizado a partir del análisis de artículos científicos, seleccionando aquellos con contenido directamente relaccionado al tema. Resultado: el Sarcoma de Kaposi fue descrito por primera vez en 1872, como un tumor mesenquimal, angioproliferativo con infiltrado inflamatorio causado por el virus herpes 8, caracaterizado por lesiones nodulares hiperpigmentadas. Hoy, es considerada la principal malignidad relacionada al Síndrome

1 Universidade Federal de Pelotas, Pelotas - Rio Grande do Sul. *E-mail: sergio.alb@hotmail.com 
de Inmunodeficiencia Adquirida y, por poder acometer cualquier sistema del cuerpo, puede comprometer importantemente la calidad de vida del portador del sarcoma. Consideraciónes finales: El sarcoma de Kaposi es una neoplasia poco prevalente, pero con el aumento de la infección por el VIH diagnosticada tardíamente, viene ganando espacio en el sistema de salud, siendo necesario saber identificarlo, pues el diagnóstico y tratamiento precoz pueden disminuir la morbimortalidad de las personas con el VIH.

Palavras-clave: Sarcoma de Kaposi, VIH, Sindrome de la inmunodeficiencia adquirida (SIDA).

\section{INTRODUÇÃO}

O sarcoma da Kaposi é uma neoplasia mesenquimal descrita pela primeira vez por Moritz Kaposi em 1872. Essa doença é causada pelo vírus herpes tipo 8 e apresenta forte associação com a Síndrome da imunodeficiência adquirida (AIDS) (TANCREDI et al., 2017). Internacionalmente, é a neoplasia mais frequente em pacientes portadores do vírus HIV e uma das principais manifestações diagnosticas da doença (GOTTLIEB et al., 1981).

A doença apresenta um tropismo por vasos sanguíneos e linfáticos da pele, mas também pode acometer outros órgãos, como pulmão e trato gastrointestinal, apresentando um grande espectro de manifestações clinicas (PIRES et al., 2018). Há quatro formas epidemiológicas com apresentações clínicos distintas: clássica, endêmica, iatrogênica e epidêmica (PELLOWSKI et al., 2014; PATROCíNIO et al., 2016). Na forma clássica da doença, o Sarcoma de Kaposi se limita as extremidades do corpo. Em pacientes com AIDS, a apresentação é sistêmica e multifocal (SANTOS et al., 2013; TIUSSI et al., 2012). A doença não se limita a uma faixa etária, podendo se manifestar em qualquer idade e com maior prevalência em homens que fazem sexo com homens. No Brasil, há mais de 800.000 pessoas com HIV/AIDS e o Sarcoma de Kaposi é a neoplasia mais frequente nesse grupo (PIRES et al., 2018).

A partir dos anos 90, por meio da terapia antirretroviral altamente eficaz, houve um declínio da morbidade e mortalidade nos pacientes portadores do vírus da imunodeficiência humana, reduzindo, por consequência, a incidência do Sarcoma de Kaposi (GOTTLIEB et al., 1981). Hoje, mesmo contando com políticas de prevenção combinada, que conjugam ações biomédicas (reduzir riscos de exposição ou transmissibilidade), comportamentais (proporcionar informação e conhecimento da percepção ou auto-avaliação do risco a exposição ao HIV) e intervenções estruturais (estratégias que visam enfrentar fatores e condições socioculturais que influenciam diretamente na vulnerabilidade de indivíduos ou grupos específicos) (MINISTÉRIO DA SAÚDE, 2017). Anualmente, uma média de 40 mil novos casos de AIDS nos últimos cinco anos, são notificados no nosso país, segundo dados da Secretaria de Vigilância em Saúde. De 1980 a junho de 2017, foram identificados 882.810 casos de AIDS no Brasil. Assim, a infecção pelo HIV/AIDS ainda é uma doença que demanda muita atenção do sistema de saúde (SECRETARIA DA SAÚDE, 2017).

Os dados acima nos mostram que a epidemia de AIDS está longe de terminar e que a mesma continua afetando a saúde dos indivíduos e o desenvolvimento das nações em muitas partes do mundo. Ao contrário da maioria das outras epidemias, o HIV afeta preferencialmente as pessoas no auge da sua vida, mesmo sendo transmissão do HIV um evento biológico, que é altamente dependente do contexto social e de suas práticas comportamentais. De acordo com Pellowski et al. (2014), a transmissão do HIV depende de quatro fatores interrelacionados: o índice de prevalência local do HIV, comportamentos individuais, fatores biológicos e condições sociais.

Embora o diagnóstico de novas infecções pelo HIV tenha declinado cerca de $11 \%$ desde 2010 , nos últimos anos tem-se notado uma maior incidência acompanhada de um diagnóstico tardio. Por consequência, a incidência dos pacientes com Sarcoma de Kaposi também aumentou, estando presente em 1,5\% do total de pacientes notificados com AIDS no período de 2003 a 2010. Em estudos regionais, a prevalência chega a números ainda mais significativos, como 9,1\% no munícipio do Rio de Janeiro (2009) e 17,1\% no estado de 
São Paulo(2010). Assim, uma doença que era pouco vista desde o início do tratamento com antirretrovirais, novamente ganha destaque na população acometida pelo HIV (TANCREDI et al., 2017).

O sarcoma é uma doença agressiva que pode implicar em complicações, como infecções oportunistas associadas a AIDS, podendo inclusive ser fatal.(PIRES et al., 2018) Ao mesmo tempo, apresenta boa resposta às diversas abordagens terapêuticas: como excisão cirúrgica, crioterapia e radioterapia, quando as lesões são localizadas e o tratamento sistêmico, reservado para o caso de lesões cutâneas de maior extensão, múltiplas ou com envolvimento de outros órgãos (PATROCíNIO et al., 2016).

Diante do que foi apresentado, o objetivo desse estudo foi revisar na literatura científicas a doença Sarcoma de Kaposi como uma condição relacionada a pacientes com HIV/AIDS e suas características, bem como descrever as principais manifestações da doença a fim de auxiliar no seu diagnóstico. Além disso, apresentar as opções de tratamento para pacientes acometidos com esta neoplasia. Para tal, o presente resumo foi realizado a partir da análise de artigos científicos e leitura analítica, selecionando, dentre os materiais encontrados, conteúdos diretamente ligados ao tema proposto

\section{RESULTADOS E DISCUSSÃO}

O HIV, vírus da imunodeficiência humana, é o retrovírus responsável por infectar células do sistema imunológico e causar a AIDS. As principais células infectados são aquelas que apresentam a molécula CD4+ em sua superfície, predominantemente os linfócitos CD4+ (linfócitos T4 ou T - Helper) e os macrófagos - a molécula CD4 age como receptor do vírus, mediando a invasão celular. O estágio tardio da infecção pelo HIV caracteriza-se por queda dos níveis dos linfócitos CD4 e o aparecimento de infecções oportunistas. A média de sobrevida após contagens de CD4 menores que 200 células $/ \mathrm{mm}^{3}$ é de 3,7 anos (EIRA, 2010).

Além da dosagem de CD4, o diagnóstico de AIDS pode ser realizado por meio da escala de sinais, sintomas e doenças baseadas no critério de Rio de Janeiro/Caracas, se resultar em um somatório maior de 10 pontos. O Sarcoma de Kaposi pontua sozinho os 10 pontos necessários para o diagnóstico (SAÚDE.GOV 2016; SAÚDE.GOV 2017).

Em 1872, Morris Kaposi descreveu uma patologia caracterizada por múltiplas lesões hiperpigmentadas, nodulares e de ocorrência mais frequente em extremidades dos membros inferiores, que posteriormente foi identificada como o Sarcoma de Kaposi. Hoje, a "forma epidêmica" da doença está diretamente relacionada com pacientes portadores do vírus HIV, sendo a forma mais agressiva e a principal doença neoplásica associada a esses pacientes (MARQUES e GARCIA, 2016). Além dessa, existem as formas "clássica", mais comum em idosos, "africana/endêmica", comum em crianças e adultos jovens negros e a "iatrogênica/imunossupressão", em pacientes transplantados (KALIL et. al, 2010).

O Sarcoma da Kaposi é um tumor mesenquimal, angioproliferativo caracterizada por angiogênese e infiltrado inflamatório, causado pelo vírus dos herpes tipo 8 (presente no núcleo das células endoteliais fusiformes do Sarcoma e responsável pela disseminação tumoral adjacente e/ou a distância no indivíduo, por meio de quimiotaxia) (LEÃO et. al, 1999), transmitido tanto por relações sexuais, quanto de forma horizontal, como pela saliva (SPODE e SCHMITT, 2003), podendo surgir em qualquer fase da infecção pelo HIV (infecção aguda, assintomática e sintomática), no entanto, a baixa contagem de células CD4+, prevê um risco aumentado para o desenvolvimento da neoplasia. Acomete, especialmente, pacientes homens na faixa etária dos 30 aos 39 anos, com práticas homossexuais ou bissexuais, com uma taxa de incidência 10 a 20 vezes maior nessa população, diferentemente do que era observado mais de duas décadas atrás, em que os principais acometidos pela doença eram homens idosos que viviam no mediterrâneo e judeus, jovens africanos ou pacientes que haviam realizado transplante (BENEDITO, 1999; COSTA et. al, 2006).

A doença, frequentemente se manifesta como tumores vinhosos, avermelhados ou rosados (quando jovens) elevados principalmente na pele, porém há também acometimento visceral, oral e ganglionar, em tecidos conectivos como ossos, cartilagens, gorduras músculos e vasos sanguíneos. As lesões de pele podem ser maculares, platiformes, papulares ou nodulares e com dimensões variadas, mas pode ocorrer 
também a elevação nódulos subcutâneos que não apresentam pigmentação visível na pele. Em negros, as lesões podem apresentar variações de coloração, podendo além da apresentação característica, serem amarronadas ou pretas. Vale ressaltar que, com o tratamento, essas lesões podem regredir especialmente no que diz respeito a seu volume, além de diminuir a intensidade de sua coloração, no entanto há relatos da permanência das lesões mesmo na ausência da doença (HIVinSite, 2018).

O acometimento da orofaringe ocorre em cerca de um terço dos pacientes e se manifesta especialmente em palato duro de forma assintomática, mas pode envolver a gengiva, língua, amígdalas, faringe e traqueia, podendo interferir diretamente na deglutição e fonação, quando acometem a mucosa oral podem ser uni ou multifocais, causando inflamação local, ulceração, sangramentos e deformação da arcada dentaria (MORALEJO et. al, 2017). Pode ocorrer acometimento do trato gastrointestinal seguido das manifestações de pele ou de forma isolada especialmente em pacientes com contagem de células CD4 muito reduzidas, podendo se manifestar de forma assintomática ou inferir alterações tanto na digestão quanto absorção dos nutrientes, que podem levar tanto a diarreia, quanto a obstrução completa do trato digestivo (SCHAWARTZ, 2004). O envolvimento de gânglios linfáticos ocorre em mais da metade dos casos e, por dificultar a drenagem linfática, promove o surgimento de linfedema principalmente em membros inferiores, periorbitais e em genitália. Geralmente, a evolução da doença tem curso crônico, podendo Inferir limitações aos indivíduos acometidos com sua progressão, já as formas letais são raras (COSTA, 2006).

Existem várias abordagens de tratamento para Sarcoma de Kaposi, visto que a doença tem inúmeras formas de apresentação, sendo necessário individualizar o tratamento para cada paciente. É indispensável iniciar o uso imediato de antirretrovirais (ARV) nos pacientes que ainda não o fazem, pois a terapia ARV está associado a reconstituição imune e controle da viremia com regressão das lesões, diminuição do número de lesões, decréscimo da incidência e aumento da sobrevida. Inicialmente, por conta da reconstituição imune, pode ocorrer piora das lesões (MANI, 2009; PASCHOAL, 2014; SULLIVAN et al, 2009).

Dentre as terapias oferecidas, pode ser realizada a radioterapia, terapia a laser ou crioterapia e injeções de vincristina ou excisão cirúrgica para doenças localizadas.(FONSECA et al, 1999; COSTA et. al, 2006) O tratamento radioterápico, é o principal método de tratamento e utiliza radiações ionizantes para destruir ou inibir o crescimento anormal das células que formam o tumor, apresentando boa resposta na regressão das lesões e efeitos colaterais (náuseas, vômitos, anemia, entre outros) que tendem a desaparecer ao longo do tratamento (AMERICAN CANCER SOCIETY, 2016). No entanto, por ser mais útil para lesões localizadas, é normalmente empregada como medida paliativa (SCHAWARTZ, 2004).

A terapia biológica (imunoterapia), conta com o interferon alfa, que foi um dos primeiros medicamentos utilizados para o tratamento do Sarcoma, age impedindo que os vírus se reproduzam e ativando o sistema imunológico para destruição viral, porém, devido a seus inúmeros efeitos colaterais e a baixa resposta ao tratamento em pacientes HIV, hoje não é mais utilizado com tanta frequência. Já a quimioterapia, utiliza medicamentos anticancerígenos (especialmente as antraciclinas) para destruir as células tumorais, sendo o principal tratamento sistêmico da doença e apresentando uma importante melhora em mais da metade dos pacientes, principalmente nos que tiveram o diagnóstico do Sarcoma de Kaposi e do HIV concomitantemente. Agem impedindo a rápida divisão celular, porém afeta tanto as células tumorais quanto as outras células do organismo, promovendo o surgimento de inúmeros efeitos colaterais como náuseas, vômitos, diarreia e aumento do número de infecções, o que torna difícil a administração de quimioterapia por longos períodos de tempo (AMERICAN CANCER SOCIETY, 2016; JUNGER, 2013). Dessa forma, uma sobrevida que antes era de menos de $10 \%$ em 5 anos, hoje, chega a cerca de $72 \%$ decorrente dos avanços dos tratamentos (AMERICAN CANCER SOCIETY, 2018).

\section{CONSIDERAÇÕES FINAIS}

O Sarcoma de Kaposi é uma neoplasia pouco prevalente e que há poucos anos era dificilmente vista pela comunidade médica. No entanto, com o aumento da infecção pelo HIV em populações com risco maior de desenvolverem esta neoplasia e com os inúmeros diagnósticos tardios da infecção pelo HIV, vem ganhando 
espaço no sistema de saúde. Além disso, evolução da doença é bastante variada podendo cursar de forma significativamente agressiva, facilitando que maioria dos pacientes falece por doenças oportunistas. Dessa forma, é necessário realizar uma anamnese e um exame físico minucioso e de qualidade, pois tanto o diagnóstico como tratamento instituídos precocemente podem diminuir a morbimortalidade das pessoas com diagnóstico de HIV/AIDS.

\section{REFERÊNCIAS}

1. AIDS.GOV. 2017. Diretrizes para organização do CTA no âmbito da prevenção combinada e nas redes de atenção à saúde. Disponível em: http://www.aids.gov.br/pt-br/pub/2017/diretrizes-para-organizacao-do-cta-noambito-da-prevencao-combinada-e-nas-redes-de-atencao. Acesso em: 01 jun. 2018.

2. AIDS.GOV 2017. O que é HIV; Sintomas e fases da aids. Disponível em: http://www.aids.gov.br/pt-br/publicogeral/o-que-e-hiv . Acesso em: 01 jun. 2018

3. American Cancer Association.2016. Disponível em: https://www.cancer.org/cancer/kaposi-sarcoma.html. Acesso em: 06 mai. 2018.

4. AMERICAN CANCER SOCIETY. 2018. Disponível em: https://www.cancer.org/cancer/kaposisarcoma/detection-diagnosis-staging/survival.html. Acesso em: 01 set. 2018.

5. BARTLETT. D.D.J.G. Medical Management of HIV Infeccion 15th Edition.

6. COSTA EL, VENANCIO MA, GAMONAL A. Sarcoma de Kaposi. HU rev, Juiz de Fora, v.32, n.3, p.77-84, jul./set. 2006

7. EIRA M. Avaliação de ateromatose subclinical em pacientes HIV/aids: determinação da velocidade de onda de pulso e da espessura media-íntima de carótidas. Biblioteca Digital de Teses e Dissertações da USP.2010; 10.11606/T.5.2010.tde-09032010-174842

8. FONSECA A.L., BOLLELA V.R, NETO R.J.P, Sarcoma de Kaposi e síndrome da imunodeficiência adquirida: características desta associação, incluindo novos conceitos sobre patogenese e tratamento. Ribeirão Preto, Simpósio: Virologia Médica I, capítulo III. 1999

9. GOTTLIEB MS, SCHROFF R, SCHANKER HM et al. Pneumocystis carinni pneumonia and mucosal candidiasis in previously healthy homosexual men: evidence of a new acquired cellular immunodeficiency. $\mathrm{N}$ Engl $\mathrm{J}$ Med 1981; 305: 1425-1431.

10. GOV.SECRETARIA DA SAÚDE. 2017. Boletim Epidemiológico, HIV/Aids 2017 - Secretaria de Vigilância em Saúde. Disponível em: https://central3.to.gov.br/arquivo/387532/. Acesso em: 01 jun. 2018.

11. HIVinSite. KROWN SE. Clinical Characteristics of Kaposi Sarcoma 2006. http://hivinsite.ucsf.edu/InSite?page=kb-00\&doc=kb-06-02-03. Acesso em: 01 jun. 2018

12. JUNGER JMB. Estudo dos doentes com Sarcoma de Kaposi após a introdução da terapêutica HAART nos últimos 19 anos no Hospital de Santa Maria - Lisboa (1994 - 2012). 2013 Sistema Integrado de Bibliotecas Repositório - Universidade de Lisboa http://hdl.handle.net/10451/11054

13. KALIL JA. ANTONIO CJ. PAPAIORDANOU F. et al. Sarcoma de Kaposi em membros inferiors: relato de caso. 2010.

14. LEÃO JC. HINRICHSEN SL. DE FREITAS BL. et al. Herpes virus humano-8 e Sarcoma de Kaposi. Revista de Associação Médica Brasileira. 1999; dx.doi.org/10.1590/S0104-42301999000100011

15. MANI D, NEIL N, ISRAEL R et al. A retrospective analysis of AIDS-associated Kaposi's sarcoma in patients with undetectable HIV viral loads and CD4 counts greater than 300 cells/mm3. J Int Assoc Physicians AIDS Care (Chic) 2009.

16. MARQUES EG, GARCIA ES. Prevalência de sarcoma de Kaposi em associação a imunodeficiência adquirida em pacientes atendidos no Hospital regional de Cáceres-MT. Revista Saberes da FAPAN. v. 3, n. 1, p. 74-81, jul./dez. 2016

17. MORALEJO B. VALLS-OTAÑÓN A. MARÍ-ROIG A. Sarcoma de Kaposi de diagnostico intraoral. 2017. Revista Española de Cirurgía Oral y Maxilofacil. dx.doi.org/10.1016/j.maxilo.2016.08.002

18. PASCHOAL EP, SANTO CCE, GOMES AMT, et al. Adesão a terapia antirretroviral e suas representações para pessoas vivendo com HIV/AIDS. Escola Anna Nery Revista de Enfermagem. Esc Anna Nery 2014;18(1):32-40

19. PATROCÍNIO J, SANTO AR, PEREIRA R, et al. Sarcoma de Kaposi clássico, a propósito de um caso clínico. Rev. Clin. Hosp. Prof. Dr. Fernando Fonseca, 2016; 4 (1/2): 45-47. http://hdl.handle.net/10400.10/1949

20. PELLOWSKI JA, KALICHMAN SC, MATTHEWS KA, et al. A pandemic of the poor: social disadvantage and the U.S. HIV epidemic. American Psychologist - Journal Article. 10.1037/a0032694 
21. PIRES CAA, NORONHA MAN, MONTEIRO JCMS, et al. Kaposi's sarcoma in persons living with HIVIAIDS: a case series in a tertiary referral hospital. An Bras Dermatol. 2018;93(4):524-8.

22. SANTOS M, VILASBOAS V, MENDES L, et al. Lymphangiectatic Kaposi's sarcoma in a patient with AIDS. An Bras Dermatol. 2013;88(2):276-8.

23. SAUDE.GOV. 2000. Aids: etiologia, clínica, diagnóstico e tratamento" Disponível em: http://bvsms.saude.gov.br/bvs/publicacoes/Aids_etiologia_clinica_diagnostico_tratamento.pdf. Acesso em: 01 jun. 2018.

24. SAÚDE.GOV. 2016. Critérios de Definição e Escala - AIDS \& DST's. Disponível em: http://www.saude.pr.gov.br/modules/conteudo/print.php?conteudo=40 Acesso em: 01 jun. 2018.

25. SAUDE.GOV 2017. Vigilância Epidemiológica da Aids e do HIV adultos e crianças. http://www.saude.sp.gov.br/resources/crt/publicacoes/arquivos-de-eventos/webconferencia-de-vigilanciaepidemiologica-do-hivaids-de-27032017/webhivaids27.03.17.pdf.

26. SCHAWARTZ RA. Kaposis's Sarcoma: An update. J Surg Oncol. 2004 Sep 1;87(3):146-51

27. SOUZA VA, SUMITA LM, FREIRE $W$ et al. Prevalence of antibodies to human herpesvirus-8 in populations with and without risk for infection in São Paulo State. Braz J Med Biol Res. 2004;37:123-7.

28. SPODE VL. SCHMITT VM. Estudo sobre as possíveis vias de transmissão do Herpesvírus Humano tipo 8. 2003. Revista da Associação Médica Brasileira. dx.doi.org/10.1590/S0104-42301999000100011.

29. SULLIVAN RJ. PANTANOWITZ L. CASPAER C. et al Epidemiology, pathophysiology and treatment of Kaposi sarcoma-associated herpesvirus disease: Kaposi sarcoma, primary effusion lymphoma, and multicentric Castleman disease. 2009; doi: 10.1086/592298

30. TANCREDI MV, PINTO VM, SILVA MH et al. Prevalence of Kaposi's sarcoma in patients with AIDS and associated factors, São Paulo-SP, Brazil, 2003-2010. Epidemiol. Serv. Saude, Brasília, 26(2), Apr-Jun 2017 10.5123/S1679-49742017000200015.

31. TIUSSI RM, CAUS ALO, DINIZ LM, et al. Sarcoma de Kaposi: clinical and pathological aspects in patients seen at the Hospital Universitário Cassiano Antônio Moraes - Vitória - Espírito Santo - Brazil. An Bras Dermatol. 2012;87(2):220-7. 